\title{
From maximal force to the field equations of general relativity
}

\author{
C. Sivaram, Arun Kenath, Christoph Schiller ${ }^{\ddagger}$
}

September 16, 2021

\begin{abstract}
We point out that field equations of general relativity are implied by a maximal force given by $c^{4} / 4 G$, analogous to the way that special relativity is implied by a maximal speed given by $c$. We present some of the arguments for this equivalence. The maximal force naturally plays the role of an elastic constant for space-time. Implications of the maximal force for gravitational wave measurements, cosmology and black holes are highlighted. Quantum aspects of the maximal force are discussed.
\end{abstract}

Keywords: general relativity, maximal force, maximal power, cosmological constant, Planck curvature.

\footnotetext{
*Indian Institute of Astrophysics, Bangalore, India, sivaram@iiap.res.in

CHRIST (Deemed to be University), Bangalore, India, kenath.arun@ christuniversity.in

Motion Mountain Research, Munich, Germany, cs@motionmountain.net
} 


\section{Introduction}

Einstein's field equations of general relativity indicate that any source of energy-momentum or energy density causes a curvature of space-time. This curvature can be specified by the Ricci tensor, for example. Here we point out that the simple existence of a maximal force value, as indicated in earlier papers [1, 2, 3], naturally leads to the field equations of general relativity. As shown in earlier work, the existence of a limiting force can be justified by invoking both Newtonian gravity and quantum theory $[1,4]$.

In the following, we provide a simple derivation of how the force limit

$$
F_{\max }=\frac{c^{4}}{4 G}=3.026(1) \cdot 10^{43} \mathrm{~N}
$$

implies the field equations of general relativity. We also discuss some related issues. We note straight away that the equivalence principle is satisfied, as the maximal force value $c^{4} / 4 G$ is independent of mass, independent of whether mass is inertial or gravitational, and is invariant, valid for every observer.

In the past, publications have proposed either $c^{4} / G$ or $c^{4} / 4 G$ as maximal force value. The oldest publications known to the authors are by Rauscher [5] and by Treder [6] and suggested the value $c^{4} / G$ as upper bound. More recent publications that focused specifically on the topic of the upper bound derived the value $c^{4} / 4 G$, including those of Gibbons [2], Schiller [3], and Barrow and Gibbons [7, 8]. Therefore we keep the factor 4 in the denominator.

\section{The field equations}

If $F_{\max }$ acts over an area $A$, the stress, or energy density, or force per unit area $\epsilon$ in the region of space covering the surface $A$ is given as:

$$
\epsilon=\frac{c^{4}}{4 G} \frac{1}{A} .
$$

For instance in the case of a spherical surface of radius $a$, the area $A=4 \pi a^{2}$ implies a uniform curvature $R=1 / a^{2}$. So,

$$
R=\frac{16 \pi G}{c^{4}} \epsilon
$$

In the general case, the contracted curvature $R / 2$ becomes the general Riemann tensor $R_{\mu \nu}$, and the energy density $\epsilon$ is described by the energy-momentum tensor $T_{\mu \nu}$ (with components of pressure, density, etc.). This yields the intermediate result:

$$
R_{\mu \nu}=\frac{8 \pi G}{c^{4}} T_{\mu \nu}
$$

The conservation of $T_{\mu \nu}$, i.e., the vanishing of its covariant derivative, dictates that $R_{\mu \nu}$ be replaced by the Einstein tensor $G_{\mu \nu}=R_{\mu \nu}-(1 / 2) g_{\mu \nu} R$ in equation (4), leading to

$$
G_{\mu \nu}=\frac{8 \pi G}{c^{4}} T_{\mu \nu}
$$


As the energy-momentum source $T_{\mu \nu}$ is a second order tensor (as known from elasticity theory), we use the second order (contracted) tensor $R_{\mu \nu}$ in equation (4). In the case that we just have a space of uniform curvature $R$, a matter density distribution $\varrho$ implies a curvature given by

$$
R=\frac{8 \pi G}{c^{2}} \varrho .
$$

A way of looking at the relation between curvature and mass-energy was provided by Sakharov [9, 10, 11]: the gravitational constant $G$, or better the maximal force, can be taken as describing the elasticity of space-time. In other words, space-time resists being curved when stress energy is applied at some point of space. So $c^{4} / 4 G$ can be interpreted as the coefficient of elasticity, relating the applied stress to the strain due to the deformation of space-time. Equations (3) to (6) confirm this description.

The similarity with elasticity can be extended further. In elastic materials, the coefficient of elasticity is, within a factor of order $\mathrm{O}(1)$, the maximal stress or maximal tension that a material can support before breaking. In gravitation, the coefficient is the maximal force that space-time can support before a horizon appears. Indeed, for black holes, the smallest radius an object of mass $M$ can have is

$$
r_{\min }=\frac{2 G M}{c^{2}} \text {. }
$$

Using the minimum diameter, this gives a maximal tension $c^{2} M / d_{\min }=c^{4} / 4 G$ - tension being energy per unit length - that space-time can support.

In superstring theory, the string tension is of the order $c^{4} / 4 G$. Also in superstring theory, when the tension or force in a physical system increases towards $c^{4} / 4 G$, space-time breaks down. It is still a subject of research to understand in detail what happens at the microscopic scale when such high force values occur.

\section{The cosmological constant}

In cosmology, we observe a cosmological constant $\Lambda$ with dimensions of a curvature that is constant throughout the universe. The cosmological constant, invoked by Einstein, implies a background stress or energy density of

$$
\varrho_{\Lambda}=\frac{\Lambda c^{4}}{8 \pi G}
$$

For the measured value of $\Lambda \approx 10^{-52} \mathrm{~m}^{-2}$, the energy density is $10^{-26} \mathrm{~kg} / \mathrm{m}^{3}$. Current observations confirm that this dark energy density presently dominates cosmic dynamics [12]. Since $\Lambda \approx 1 /\left(4 \pi R_{H}^{2}\right)$, where $R_{H}$ is the Hubble radius, the expression $1 / \Lambda$ gives the surface area of the Hubble sphere. Thus the maximal force, distributed over the area of the Hubble sphere, gives the density of dark energy.

So, if we have a general background curvature $\Lambda$ in the universe, in addition to local sources, the term (8) has to be added in equation (4), thus giving

$$
G_{\mu \nu}+\Lambda g_{\mu \nu}=\frac{8 \pi G}{c^{4}} T_{\mu \nu}
$$


where as usual $g_{\mu \nu}$ is the metric tensor of space-time, whose derivatives give the curvature.

In short, general relativity's field equations, with the cosmological constant, emerge from postulating a maximal force in nature.

\section{Black holes}

The maximal force also implies a minimal diameter for a mass $M$ given by $d=4 G M / c^{2}$. This diameter defines a black hole. So, black holes are predicted by the postulate of maximal force. They have an escape velocity given by $c$. Indeed, both Michell and Laplace had deduced the possibility of black holes in the case that the escape velocity from an object of mass $M$ becomes $c$.

The maximal force $c^{4} / 4 G$ is closely related to horizons. Approaching mass to a back hole whatever the size of the black hole or of the mass - yields a gravitational attraction that is never larger than $c^{4} / 4 G$, even in general relativity [2]. In fact, the value $c^{4} / 4 G$ is the largest possible force between two black holes. This can be seen even in the Newtonian limit. Using the Newtonian expression $F=G M^{2} / r^{2}$ with the smallest possible radius $r=2 G M / c^{2}$ for any mass $M$ yields $F_{\max }=c^{4} / 4 G$.

In short, maximal force arises at horizons. Indeed, there appears no way to achieve maximal force without the help of a horizon. At the same time, the rarity of horizons in everyday life is the reason that the maximal force is not of experimental relevance.

Maximal force allows deducing further limits. The maximal electric charge $Q$ possible on a body of mass $M$ can be estimated from $G M^{2} / r^{2}=Q^{2} / r^{2}$ to be $Q=\sqrt{G} M$. Similarly, the maximal possible angular momentum of a body is $J=G M^{2} / c$. Related limits on inertia, magnetic moment and related quantities also follow from maximal force $[8,13]$.

\section{Quantum theory and quantum gravity}

The maximal force can also be justified using quantum theory. A quantum particle with mass $m$ cannot be localised to within a distance less than $\hbar / m c$. This implies a maximal acceleration given by [14]

$$
a_{\max }=m c^{3} / \hbar
$$

and, likewise, a maximal force of $m^{2} c^{3} / \hbar$. The maximal value for the mass $m$ of a quantum particle is the Planck mass, corresponding to a localization of a Planck length. Using the definition of the corrected Planck mass $m^{2}=\hbar c / 4 G$, we recover $F_{\max }=c^{4} / 4 G$.

Maximal force implies limits for many physical observables. For example, maximal force, together with the quantum of action, leads to a minimal distance $L_{\mathrm{Pl}}=\sqrt{4 \hbar G / c^{3}}$ and a maximal curvature $1 / L_{\mathrm{Pl}}^{2}$. Maximal force thus implies the lack of singularities.

We can also get some insight into quantum gravity. If we rewrite the Hilbert action $I$ using $\hbar$ as $I=n \hbar$, where $n$ is a natural number, we get

$$
\frac{1}{c} \times \frac{c^{4}}{\hbar G} \int R d^{4} x=n=\frac{I}{\hbar}
$$


The left hand side now contains the Planck curvature $R_{\mathrm{Pl}}=c^{3} / \hbar G$, and the maximal force $c^{4} / G$ is naturally incorporated in the gravitational action. This shows that the maximal force and the Planck length are deeply related.

In the quantum domain, limits that contain the quantum of action lead to uncertainty relations. In particular, the Hilbert action suggests an uncertainty relation for curvature and volume given by

$$
\Delta R \times \Delta V \gtrsim L_{\mathrm{Pl}}
$$

In other terms, curvature $R$ and volume $V$ form conjugate variables. If the volume is large, curvature fluctuations are small. If the volume is small, of the order of the Planck volume, then the fluctuations in curvature are large, of the order of the Planck curvature $L_{P l}^{-2}$. This in turn suggests the uncertainty relation $\Delta x \times \Delta y \gtrsim L_{\mathrm{Pl}}^{2}$.

In short, the maximal force limit is independent of quantum theory but deeply related to it. Combining maximal force with quantum theory leads to the main uncertainty relations of quantum gravity.

\section{Maximal power}

The maximal force also leads to a maximal power or maximal luminosity $P_{\max }=c^{5} / 4 G \approx$ $9.1 \cdot 10^{51} \mathrm{~W}$ from a localized source, or about 50700 solar masses per second. [15, 16, 17]. This limit can be tested. An example is a source of gravitational waves. As the waves propagate over a large distance, the curvature distortion becomes small. The gravitational wave amplitude, the strain $h$, is given by [10]

$$
h \approx \frac{G E}{c^{4} r},
$$

where $E$ is the gravitational wave energy at source and $r$ is the distance from the source. Again, maximal force appears in the expression. The expression for the strain is thus

$$
h \approx \frac{\text { Energy of gravitational wave at source }}{r \times \text { maximal force }} .
$$

This expression can be tested. Suppose that in black hole merger, 10 solar masses are converted into energy of gravitational waves, thus $E \approx 10^{48} \mathrm{~J}$. Then the strain at a distance of $10^{25} \mathrm{~m}$ would be $h \approx 10^{-21}$, which is within the LIGO limit. The ratio $E / r$ gives the 'force' exerted by gravitational waves with initial energy $E$ from a source at a distance $r$. In other words, the strain

$$
h \approx \frac{E}{r \times F_{\max }}=\frac{F}{F_{\max }}=\frac{\Delta l}{l}
$$

can be used to estimate the power $P$ at the source of gravitational waves. Such measurements are important, because black holes mergers are the energy sources with the highest luminosity or power that are known. So far, all measurements confirm that the maximal force or maximal power are never exceeded [18, 19]. In fact, the highest instantaneous power ever measured is about $0.5 \%$ of the maximal value. 


\section{Consequences for cosmology}

The maximal power, $c^{5} / 4 G$, introduced above as a natural consequence of maximal force has direct consequences for cosmology [20]. To see this, consider an expanding universe, where the maximal power is continuously generated (for a time $t$ ), so the total energy generated is $\frac{c^{5}}{G} t$. During this time, the universe has expanded to a volume $(4 \pi / 3) c^{3} t^{3}$. So the energy density filling this volume is

$$
\epsilon=\frac{3 c^{5} t}{4 \pi G c^{3} t^{3}} .
$$

This maximal possible energy density of the radiation within the volume can be equated to the Stefan-Boltzmann relation $\epsilon=a T^{4}$, with $a$ being the Stefan-Boltzmann constant. This gives

$$
T=\left(\frac{3 c^{2}}{4 \pi a G}\right)^{1 / 4} \frac{1}{\sqrt{t}} .
$$

The same relation appears in the usual big bang picture. The temperature at the present time is predicted to be around $40 \mathrm{~K}$, not too far from observations for such a simple argument.

Dividing the maximal power $c^{5} / 4 G$, integrated over the age $t_{0}$ of the universe, by the Hubble volume, yields an upper limit on baryon mass density $\varrho_{\mathrm{b}, \max }$ given by

$$
\varrho_{\mathrm{b}, \max }=\frac{3}{16 \pi G\left(t_{0}\right)^{2}}=4.7 \cdot 10^{-27} \mathrm{~kg} / \mathrm{m}^{3} .
$$

This is half the critical density. The observed baryon density is about 10 times smaller. Also cosmological observations do not contradict the power limit.

\section{Locality}

To get used to maximal force, the similarity with statements about maximal speed is often helpful. For example, the limit on observable physical speed $c$ is only valid locally. Similarly, the limit on observable physical force $c^{4} / 4 G$ is only valid locally. Locality is an essential aspect in these statements. Maximal force is a local concept like maximal speed $c$ in special relativity.

Any observer can add speeds of separate, distant objects and find a sum exceeding the local limit $c$. In the same way, any observer can add forces acting between separate, distant mass configurations and find a sum exceeding the local limit $c^{4} / 4 G$. For example, $c^{4} / 4 G$ can be exceeded if many black holes merge, or if the electrostatic force inside atoms is added for a sufficiently large number of atoms. This mistake was also made in statements in references [3] and [21], where it was wrongly claimed that maximal force is valid for any physical surface. This is not correct; the maximal force only applies locally. Also a recent preprint by Jowsey and Visser [22] overlooked the locality requirement. In their calculations of equatorial forces, the authors have added forces acting at different locations and found a sum larger than $c^{4} / 4 G$. In contrast, whenever one calculates (or measures) local forces, i.e., forces at a single location in space-time, the values are never larger than $c^{4} / 4 G[23]$. 
Maximal force is a local limit valid universally. For any force measured locally, the maximal value is valid at every point in space-time. In a general space-time, curvature varies from point to point. The stress on space varies in the same way. The stress has a local value, defined for any infinitesimal area $R_{a b} d x^{a} d x^{b}$ of a curved surface. The gravitational constant $c^{4} / 8 \pi G$ plays the role of an elastic constant. The field equations hold globally and are generally covariant; the limit $c^{4} / 4 G$ is invariant and valid everywhere, at all times.

\section{Dependence of force on the frame of reference}

The maximal force and the maximal acceleration mentioned above are measured in the proper frame. Can the maximal values be exceeded by changing to another frame of reference, with a relative velocity $v$ to the proper frame?

The transformation properties of force and acceleration were deduced from special relativity long ago [24]. The simplest situation occurs when acceleration and relative velocity are parallel. In this case, the proper acceleration $a_{0}$ and the acceleration $a_{v}$ are related by

$$
a_{0}=\gamma^{3} a_{v}
$$

As usual, the Lorentz factor $\gamma$ is given by $\gamma=\left(1-v^{2} / c^{2}\right)^{-1 / 2}$ and is always larger than 1 . The value of $a_{v}$ in another frame of reference is thus never larger than $a_{0}$, the value in the proper frame. A more complex expression is valid in the general case, when $a$ and $v$ are not parallel; nevertheless, $a_{0}$ remains the highest possible value. This applies both to 3 -acceleration and to 4-acceleration.

In the case of force, the transformation relations show that in a frame with relative velocity $v$, the parallel component remains unchanged and the other components are reduced by a factor $\gamma$ [24]. Again, the proper force is never exceeded by moving to another frame of reference. In short, coordinate transformations do not allow exceeding maximal force.

\section{Further counter-arguments}

The maximal force is the maximal gravitational force between two black holes. One can then ask whether two black holes with opposite electric charges attract even more strongly. The answer is no. Because all interactions lead to curvature, charged black holes simply have a smaller horizon radius than uncharged ones; but the maximal force holds independently of the radius of the horizon and independently of the interaction that is involved in generating the horizon. The argument also applies to combinations of gravitational and nuclear interactions. In conclusion, even though in everyday life gravity is the weakest interaction, gravity nevertheless provides an upper limit to all forces.

It is sometimes suggested that gravity is not a force in general relativity. This is of no concern in the present context; the value $c^{4} / 4 G$ provides a limit for any locally measurable momentum 
change $d p / d t=F$. Momentum change is commonly called force, and the maximal momentum change is independent of the type of interactions involved.

\section{Discussion and conclusion}

The concept of local maximal force $c^{4} / 4 G$ contains and implies spatial curvature, horizons, and black holes. Local maximal force also contains Einstein's field equations.

Attempts to exceed the maximal force are not successful, neither in real experiments nor in thought experiments. If they were, general relativity would be falsified, and an alternative theory of gravitation would be needed. Various alternative theories indeed allow exceeding maximal force [25].

The validity of the force limit $c^{4} / 4 G$ is equivalent to general relativity and allows describing gravitation in a simple and intuitive manner.

\section{References}

[1] V. de Sabbata and C. Sivaram, On limiting field strengths in gravitation, Foundations of Physics Letters 6 (1993) 561-570.

[2] G.W. Gibbons, The maximum tension principle in general relativity, Foundations of Physics 32 (2002) 1891-1901.

[3] C. Schiller, General relativity and cosmology derived from principle of maximum power or force, International Journal of Theoretical Physics 44 (2005) 1629-1647.

[4] V. de Sabbata and C. Sivaram, Maximum acceleration and magnetic field in the early universe, Astrophys. Space Sci. 176 (1991) 145-148.

[5] E.A. Rauscher, The Minkowski metric for a multidimensional geometry, Lettere al Nuovo Cimento 7 (1973) 361-367.

[6] H.-J. Treder, The planckions as largest elementary particles and as smallest test bodies, Foundations of Physics 15 (1985) 161-166.

[7] J.D. Barrow and G.W. Gibbons, Maximum tension: with and without a cosmological constant, Mon. Not. Roy. Astron. Soc. 446 (2014) 3874-3877.

[8] J.D. Barrow and G.W. Gibbons, Maximum magnetic moment to angular momentum conjecture, Phys. Rev. D 95 (2017) 064040.

[9] A.D. Sakharov, Vacuum quantum fluctuations in curved space and the theory of gravitation, Sov. Phys. Dokl. 12 (1968) 1040-1041.

[10] K.S. Thorne, C.W. Misner and J.A. Wheeler, Gravitation, Freeman (1973).

[11] C. Sivaram, Uncertainty principle limits on the cosmological constant, International Journal of Theoretical Physics 25 (1986) 825-827.

[12] A. Kenath, S.B. Gudennavar, C. Sivaram, Dark matter, dark energy, and alternate models: A review, Advances in Space Research, 60 (2017) 166-186. 
[13] V. de Sabbata and C. Sivaram, The central role of spin in black hole evaporation, in Black Hole Physics (NATO ASI Series 364) (Kluwer Academic, Dordrecht, 1992), p. 225.

[14] E.R. Caianiello, Maximal acceleration as a consequence of Heisenberg's uncertainty relations, Lettere al Nuovo Cimento 41 (1984) 370-372.

[15] C. Sivaram, K. Arun and L. Rebecca, Planckian pre big bang phase of the Universe, Astrophys. Space Sci. 365 (2020) 17.

[16] C. Sivaram, A general upper limit on the mass and entropy production of a cluster of supermassive objects, Astrophysics and Space Science 86 (1982) 501-504.

[17] V. Cardoso, T. Ikeda, C.J. Moore and C.-M. Yoo, Remarks on the maximum luminosity, Physical Review D 97 (2018) 084013.

[18] LIGO Scientific Collab. and Virgo Collab., GWTC-1: A gravitational-wave transient catalog of compact binary mergers observed by LIGO and Virgo during the first and second observing runs, Phys. Rev. X 9 (2019) 031040.

[19] LIGO Scientific Collaboration and Virgo Collaboration, Properties and astrophysical implications of the 150 Msun binary black hole merger GW190521, Astrophysical Journal Letters 900 (2020) L13, preprint at www.arXiv.org/abs/2009.01190.

[20] V. de Sabbata and C. Sivaram, A minimal time and time-temperature uncertainty principle, Found. Phys. Lett. 5 (1992) 183-189.

[21] C. Schiller, Maximum force and minimum distance: physics in limit statements, preprint at www.arXiv.org/abs/physics/0309118.

[22] A. Jowsey and M. Visser, Counterexamples to the maximum force conjecture, preprint at www.arXiv.org/abs/2102.01831.

[23] C. Schiller, Comment on "Maximum force and cosmic censorship" Physical Review D 104 (2021) 068501.

[24] C. Møller, The Theory of Relativity (Clarendon Press, 1952).

[25] M.P. Dabrowski and H. Gohar, Abolishing the maximum tension principle, Physics Letters B 748 (2015) 428-431. 